Reba Patricia. Role of food and nutrition and prevention in pathogenesis of the arterial occlusive disease. Journal of Education, Health and Sport. 2020;10(3):120-125. eISSN 2391-8306. DOI http://dx.doi.org/10.12775/JEHS.2020.10.03.012

https://apcz.umk.pl/czasopisma/index.php/JEHS/article/view/JEHS.2020.10.03.012

https://zenodo.org/record/3726428

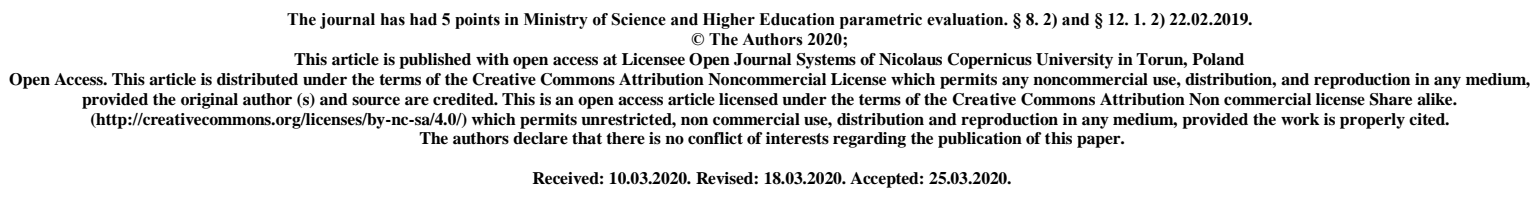

\title{
Role of food and nutrition and prevention in pathogenesis of the arterial occlusive disease
}

\author{
Patricia Reba
}

Faculty of Medicine and Health Sciences, Jan Kochanowski University, Kielce, Poland

\begin{abstract}
Admission

Atherosclerosis is a chronic inflammatory disease of complex etiopathogenesis. Improper diet, genetic predisposition, lack of physical activity, and smoking are just a few of the major factors contributing to the development of atherosclerotic plaque. Diet in atherosclerosis, treatment is based. Antiatherosclerotic diet is one of many components, which should provide not only the treatment of such a state, but primarily prevention. Accordingly, balanced nutrition regimen can reduce cholesterol synthesis in the liver, which contributes to the development of atherosclerosis. Limiting the intake of trans fatty acids and saturated fatty acids allows for inhibiting the development of atherosclerotic plaque.
\end{abstract}

Aim

The aim of the study is to analyze the role of food and nutrition in the prevention and pathogenesis of atherosclerosis of the lower extremities.

Material and methods

A review of available literature. 
Results

Adequate intake of potassium in the diet contributes significantly to reducing the risk of atherosclerosis and hypertension. Potassium determines many important functions in the body. Due to the fact that the monounsaturated fatty acids reduce LDL cholesterol levels, the proportion should be in the diet increased to $15 \%$ and for the secondary prevention of up to $20 \%$. In the prevention of atherosclerosis is very important to maintain an appropriate ratio of omega- 6 to omega- 3 ratio is absolutely should be no more than 4. It is extremely important in the diet because distorted value for the benefit of omega- 6 favors the formation of atherosclerotic lesions. respective supply of carbohydrates also has a huge impact on the development of atherosclerotic lesions. The recommended amount of anti-atherosclerotic dietary is up to $60 \%$ provided that the people do not apply to such ailments as hypertension, overweight or obesity, elevated fasting blood sugar levels, decreased HDL cholesterol and elevated triglycerides.

\section{Conclusions}

General nutritional recommendations play the role of a signpost, which you should use expecting to improve health. It should be noted, however, that is not all. Nutritional prevention is significant, but equally important is lifestyle modification. Atherosclerotic diet is an important part of the treatment of the disease and prevent further growth of atherosclerotic plaque. Atherosclerosis requires the use of low-fat diet that is necessary to beat high blood cholesterol levels.

Key words: food; nutrition; arterial occlusive disease.

\section{Admission}

Atherosclerosis is a chronic inflammatory disease of complex etiopathogenesis. it's a diseasetouching the inside of blood vessels. Improper diet, genetic predisposition, lack of physical activity, and smoking are just a few of the major factors contributing to the development of atherosclerotic plaque. The progression of fibrosis, which consists primarily of calcium and oxidized forms of fat makes the width of the interior of the vessel becomes smaller. Diet in atherosclerosis, treatment is based. Pharmacological agents will not bring the expected results if accepting them, still will consume large amounts of processed and rich in fats unfavorable product. Diet can effectively inhibit the development of the disease, which mainly cause calcium deposits mixed with macrophages and fibrin (the so-called. Atherosclerotic plaquewa), which is cemented and blocks the lumen of blood vessels. Antiatherosclerotic diet is one of many components, which should provide not only the treatment of such a state, but primarily prevention. Accordingly, balanced nutrition scheme allows to reduce cholesterol synthesis in the liver, which contributes to the development of atherosclerosis. Limiting the intake of trans fatty acids and saturated fatty acids allows for inhibiting the development of atherosclerotic plaque. Inclusion of unsaturated fatty acids in the diet fat contributes to the reduction of existing deposits. Dissemination of healthy nutrition is an important method for the primary prevention of diseases caused by atherosclerosis $[1,5]$. Achieving the right weight is diametric importance in the prevention of atherosclerosis. Losing excess weight determines:

- reducing the occurrence of inflammation in the body,

- reduction of total cholesterol and LDL.

- lower blood pressure,

- better blood circulation $[2,5]$. 
Due to the, Monounsaturated fatty acids that reduce LDL cholesterol levels, the proportion should be in the diet increased to $15 \%$ and for the secondary prevention of up to $20 \%$. Such values can be achieved by adding olive oil to cold dishes or oil Rape, eating hazelnuts and almonds, which are a rich source of oleic acid or cashew nuts, pumpkin seeds, or avocado. In the prevention of atherosclerosis is very important to maintain an appropriate ratio of omega- 6 to omega- 3 ratio is absolutely should be no more than 4 . It is extremely important in the diet because distorted value for the benefit of omega- 6 favors the formation of atherosclerotic lesions. Omega 3 exhibit:

- anticoagulants,

- anti-inflammatory,

- inhibit shrinkage of blood vessels.

- have a beneficial effect on the lipid profile lowering total cholesterol and triglyceride levels. - lower blood pressure [3,4,5].

A rich source of omega 3 are oily fish of the sea, they should be consumed 2 times a week. In order to cover the daily requirement of omega 3 just approx. 30 - 40 grams smoked mackerel, or approx. 85 grams of salmon. Another source of omega 3 are flaxseed oil, but only for the short period of validity ( 3 - 4 months), walnuts, and flaxseed. Avoid fats which in its composition to contain a large amount of saturated fats and trans fatty acids [5].

To limit the amount of saturated fatty acids should be limited consumption of products such as:

- butter, lard, fat;

- meat, especially fatty pate, salami, ham fat, all kinds of sausages, fuet;

- meat with visible fat;

- milk, yogurt greater than $2.0 \%$ fat, fat cottage cheese;

- cheese $[4,5]$.

Intake of foods containing unsaturated fatty acids trans isomers results:

- increase in the level of "bad" cholesterol,

- reducing the level of "good" HDL

- impaired insulin response to an increase in blood glucose (this may This resulted in insulin resistance in the future)

- the severity of the degenerative processes by increasing the number of free radicals in the body $[3,7]$.

This is conducive to the accumulation of cholesterol in the arteries and can lead to atherosclerosis. A rich source of these acids are:

- fully or partially hydrogenated vegetable oils present in the mixes various types of spreads, margarines, particularly hard, inferior quality;

- the products repeatedly fried in the same oil eg. French fries, fried burgers with burgers, pies of frying, breaded and deep fried pork (a multiple by frying in oil form harmful isomers of fatty acids, which are excreted in the product);

- pastry e.g. .: French pastries, donuts etc. (used in their manufacture fats are a source of transisomers) $[4,8]$.

Type of carbohydrates is essential in the use of anti-atherosclerotic diet. Recent studies show that the supply of suitable carbohydrates also has a huge impact on the development of atherosclerotic lesions. The recommended amount of dietary anti-atherosclerotic up to $60 \%$ 
provided that a person shall not apply to such ailments as hypertension, overweight or obesity, elevated fasting blood sugar levels, decreased levels of cholesterol HDL, and high levels of triglycerides. In the presence in an individual one of the above problems, this amount is reduced. If you are in someone 3 of 5 aforementioned signs, it means that the person is suffering from tin. "metabolic syndrome." Therefore, you should limit the intake of carbohydrates to 50\%. If problems with the atherosclerosis already exists, the individual is suffering from any disease from cardiovascular or has an elevated blood cholesterol levels blood, then apply the recommendations for secondary prevention, ie, carbohydrate intake should be up to 50-55\% energy from the diet. This limitation is the result of adverse effect of easily digestible carbohydrates with a high glycemic index. Occur mainly in:

- white, wheat bread;

- all kinds of sweet buns, French cakes, cookies, sweet pastries;

- sweet juices and soft drinks;

- ripe fruits;

- flavorless white rice thin pasta, French fries, potato pure [7,8].

Excessive consumption of these products causes:

- rapid and high increase in blood sugar level, which can lead over time to weaken the action of insulin and deregulation system insulin-glucagon responsible for regulating blood glucose level in combination with overweight and obesity, excessive intake of these products may lead to insulin resistance or diabetes type 2;

- an increase in blood clotting;

- increase in the levels of triglycerides;

- decline the level of beneficial HDL cholesterol [4,7,8].

Therefore, their participation in the recommended diet should be no more than $10-20 \%$ of total energy, which is less than $30 \%$ of energy from carbohydrates. The rest (30-45\% of total energy, which is above $70 \%$ of energy from carbohydrates) should come from carbohydrates so. complex, difficult digestible, low glycemic index which occur in thick groats (pearl barley, barley, buckwheat), semi-hard pasta, rice brown, parabolic, Basmati dark wholemeal bread, multigrain bread. It is therefore appropriate to eat dark bread, and as additives for second courses used cereal, rice parboiled, but dente pasta. Easily digestible carbohydrates should be consumed mainly in the form of fruits, which due to the high content of vitamins,

Dietary fiber has a beneficial effect such as reduction of weight gain leachabletion toxins and by-products of metabolism in the gastrointestinal tract, stability of blood glucose levels (preventing diabetes). Additionally, the soluble fraction has a particularly advantageous effect in combating and prevention of atherosclerosis. to the reaction outcome include pectins, gums, mucilages, $\beta$-glucans which bind cholesterol in the gel structure and enhance its excretion, causing a decrease in total cholesterol and LDL blood. These fractions are mainly in vegetables, for example. Broccoli, spinach, carrot, seeds of leguminous plants (peas, beans, lentils), and fruits, for example. plums, apples, pears, cereals barley or oats (flakes). It is important that while consumption of fiber, do not forget to drink large quantities of water because of fact, the fibers in the colon absorbs the water and the insufficient amount may result in unpleasant bowel movements or constipation. Due to the beneficial properties of fiber should consume at least 20 grams of fiber per day, which is, for example. approx. 50 
grams of dried plums pitted (average handful) a juice, raspberry multivegetable and glass or approx. 1.5 pouch buckwheat or approx. 4 slices of bread wholemeal rye $[5,7,8]$.

Antioxidants anti-free radicals exhibit. This means that degenerative and inactivate harmful effects of free radicals. This is particularly important due to the fact that an excess of free radicals induced, for example. Antioxidant stress leads to damage of the vascular endothelium. This favors the accumulation of cholesterol and other substances on the walls of arteries, which in turn increases the risk of incidents related health limb ischemia. In particular, vitamin $\mathrm{E}$ (alpha tocopherol), provitamin $\mathrm{A}$ (beta-carotene) and vitamin $\mathrm{C}$ (ascorbic acid) contained in vegetables, fruit and vegetable oils, remove excess free radicals, preventing their adverse effects on the body. According to the recommendations of the pyramid rational nutrition is recommended at least 400 grams of fruit and vegetables a day, wherein the prevention of atherosclerosis should be even greater amount. It is recommended to consume 1 - 2 servings of fruit and $3-4$ vegetables a day (1 serving is eg. A glass of raspberries, strawberries, a medium-sized apple, pear, plums and bunch eg. The average size of tomatoes, peppers half high, glass cooked broccoli etc .) $[1,2,8]$.

Phytosterols and phytostanols are substances known for their beneficial properties anti- atherosclerotic. Partially inhibit the intestinal absorption of cholesterol, thereby lowering its supply from the diet. Sterols are naturally occurring mostly in products of plant origin, such as nuts, vegetable oils, fruits and vegetables. While stanols are naturally present in minute quantities, and are mainly manufactured by a suitable manufacturing process (hydrogenation sterols). Those skilled in the art recommend feed intake of 2 grams of stanols and sterols per day to reduce cholesterol levels. It has been shown that this quantity lowers LDL cholesterol by approx. 10\% [8].

lycopene It belongs to the group of carotenoids and is considered to be one of the stronger antioxidant, thereby protecting against free radicals. Additional effects of lycopene include:

- inhibition of the synthesis of cholesterol in the body,

- facilitating the removal of the "bad" cholesterol, LDL [8].

The main source of lycopene in the diet are tomato and dairy: tomato purees, tomato juice, tomato concentrates, pasta sauces, ketchup, wherein the lycopene is absorbed better than the tomato products of the same tomato. It also occurs in small quantities in some fruit: watermelon, red grapefruit $[7,8]$.

Adequate intake of potassium in the diet contributes significantly to reduce the risk of atherosclerosis and hypertension. Potassium determines many important functions in the body:

- lowering blood pressure and preventing hypertension,

- regulates heart rhythm,

- by activation of the enzyme ATPase, it helps to provide energy for the heart amplifying them,

- inhibits the formation of blood vessels free radicals,

- it acts anticoagulation, decreases blood viscosity and the tendency to clot.

- it is a component of body fluid, improves the oxygen supply to the brain,

- is necessary to normal muscle and nerve conduction [6]. 
A good source of potassium in the diet are potatoes, much of this element shall also include: almonds, walnuts, dried apricots, bananas, broccoli and tomatoes [6].

Folate regulate blood levels of homocysteine (including vitamin B12 and B6). Homocysteine has been recognized as a direct factor in the increased risk of cardiovascular disease. High content of folate include beans, soybeans, lentils, peas, green leafy vegetables (lettuce, spinach, beet leaves), broccoli, Brussels sprouts, pork or chicken liver [7,8].

General nutritional recommendations play the role of a signpost, which you should use expecting to improve health. It should be noted, however, that is not all. Nutritional prevention is significant, but equally important is lifestyle modification.

\section{References}

1. Eurodiet Core Report. Nutrition and diet for healthy lifestyles in Europe: Science and policy implications. Publ. Health Nutr., 2001, 4 (2A), 265-252

2. Bronte-Stewart B .: The effect of dietary fats on the blood lipids and Their relation is ischemic heart disease. Brit. Med. Bull. 1958, 14, 243-252

3. Mozaffarian D, Katan MB, Ascherio A., et al .: Trans fatty acids and cardiovascular disease. N. Engl. J. Med., 2006, 1601-1613

4. Gardner Ch.D., Coulston A. Chatterjee L., et al .: The effect of a plant-based diet on plasma lipids in hypercholesterolemic adults. Ann. Intern. Med., 2005, 142, 9, 725-733

5. Szostak WB, A .: CichockaMediterranean diet in the prevention of cardiac. The monograph for physicians. Via Medica. gdańsk 2009

6. WB Szostak, Jarosz, M .: The Influence of salt intake on the development of cardiovascular disease. Alive. All leading. Metab., 2010, 37, 5-6, 374-380

7. Kłosiewicz-Latoszek L., Szostak WB, Podolec P. et al .: Prevention Forum Polish Guidelines on the rules for proper nutrition. In: Handbook Polish Forum for Prevention, Vol.

2. Edited. P. Podolca. Med. Prakt. Kraków 2010

8. http://www.izz.waw.pl/ [13.03.2020] 